Chronic Obstructive Pulmonary Diseases:

Journal of the COPD Foundation

COPD

rovuatition

\title{
Review
}

\section{Alpha-1 Antitrypsin Deficiency-Mediated Liver Toxicity: Why Do Some Patients Do Poorly? What Do We Know So Far?}

Marion Bouchecareilh, $\mathrm{PhD}^{1}$

\begin{abstract}
Alpha-1 antitrypsin deficiency (AATD) is a rare genetic disease caused by mutations in the SERPINA 1 gene and is associated with a decreased level of circulating alpha-1 antitrypsin (AAT). Among all the known mutations in the SERPINA 1 gene, homozygous for the $\mathrm{Z}$ allele is well-known to result in both lung and liver disease. Unlike the lung injury that occurs in adulthood with the environment (notably, tobacco) as a co-factor, the hepatic damage is more complicated. Despite a common underlying gene mutation, the liver disease associated with AATD presents a considerable variability in the age-of-onset and severity, ranging from transient neonatal cholestasis (in early childhood) to cirrhosis and liver cancer (in childhood and adulthood). Given that all the cofactors-genetics and/or environmental- have not been fully identified, it is still impossible to predict which individuals with AATD may develop severe liver disease. The discovery of these modifiers represents the major challenge for the detection, diagnosis, and development of new therapies to provide alternative options to liver transplantation. The aim of this current review is to provide an updated overview of our knowledge on why some AATD patients associated with liver damage progress poorly.
\end{abstract}

Abbreviations: alpha-1 antitrypsin deficiency, AATD; alpha-1 antitrypsin, AAT; endoplasmic reticulum, ER; ER-associated degradation, ERAD; ubiquitin-proteasome system, UPS; inclusion bodies, IBs; periodic acid-Schiff, PAS; unfolded protein response, UPR; body mass index, BMI; hepatocellular carcinoma, HCC; heat shock response, HSR; jun-N-terminal kinase, JNK; heptacyte nuclear factor 4 alpha, HNF$4 \mathbf{a}$

Funding Support: not applicable

Date of Acceptance: December 11, 2019

Citation: Bouchecareilh M. Alpha-1 antitrypsin deficiency-mediated liver toxicity: why do some patients do poorly? What do we know so far? Chronic Obstr Pulm Dis. 2020;7(3):172-181. doi: https://doi.org/10.15326/jcopdf.7.3.2019.0148

1 National Institute of Health and Medical Research (INSERM), National Center for Scientific Research (CNRS), University Bordeaux, Bordeaux Research In Translational Oncology, BaRITOn, Bordeaux, France

\section{Address correspondence to:}

Marion Bouchecareilh, $\mathrm{PhD}$

INSERM U1053 BaRITon

Bat 1 A 2 eme étage

146, Rue Léo Saignat

33076 Bordeaux, France

Phone: +33(0)557571607

Email: marionb@ibgc.cnrs.fr / marion.bouchecareilh@inserm.fr

\section{Keywords:}

alpha-1 antitrypsin deficiency; liver disease; modifiers; genetic factors

\section{AATD-Mediated Liver Disease: General Introduction}

Alpha-1 antitrypsin deficiency (AATD), is a rare, inherited disorder that affects approximately 1 in 2000-5000 births in the white population ${ }^{1}$ and is associated with lung (emphysema) and/or liver damage (cirrhosis). Mutations in the SERPINA 1 gene that encodes the alpha-1 antitrypsin (AAT) protein cause this deficiency, leading to a reduced level of AAT in serum. ${ }^{2,3}$ The normal SERPINA 1 allele is referred to as $\mathrm{M}$ allele and more than 150 SERPINA 1 mutants have been described to date. Nevertheless, only a few of these mutants mediate liver disease. Among 
all those variants, the $\mathrm{Z}$ allele is the most frequent variant associated with liver disease. ${ }^{4}$ It is caused by a point mutation, a single amino acid substitution (Glu342Lys), which results in an aberrantly folded protein. ${ }^{1,5}$ Unlike the wild-type (WT) AAT protein secreted by the hepatocytes (primary and major site of production of AAT), the $\mathrm{Z}$ mutant folds inefficiently and never reaches the trafficking and secretion pathway. Consequently, homozygous ZZ patients have approximately only a $10 \%-15 \%$ circulating level of AAT compared to the WT.

The $\mathrm{Z}$ variant mutation leads to the retention of the $\mathrm{Z}$ protein within the hepatocyte endoplasmic reticulum (ER), in both soluble and aggregate forms. Following translation and entry into the ER, the soluble nascent Z-AAT protein is supported by the ER quality control system. Subsequently, the ER clearance pathwayER-associated degradation (ERAD) -targets the mutant to the ubiquitin-proteasome system (UPS) for degradation. ${ }^{6}$ Several studies suggest insufficient clearance of Z-AAT results in further retention followed by $\mathrm{Z}$ aggregation. ${ }^{7,8}$ These aggregates are packed into hepatocyte inclusion bodies (IB) (Figure 1). These structures are the histopathological hallmark of AATD liver disease on biopsy and stain positively in hepatocytes with periodic acid-Schiff (PAS) after treatment with diastase (Figure 1). The mechanism of IB formation and their heterogeneity in the distribution and size within hepatocytes (Figure 1) are not clearly understood and elucidated.

The retention and accumulation of the $\mathrm{Z}$ aggregates in the ER present a gain-of-function toxic effect leading to hepatocyte toxicity and initiating events in the pathophysiology of liver disease. ${ }^{9}$ Based on this idea, preventing production and/or accumulation of Z-AAT was shown to reverse liver injury in disease models. ${ }^{10,11}$ The concept of Z-AAT gain of toxic function has been supported by the recent findings of Clark et al. ${ }^{12}$ The authors have evaluated the relationship between positive PAS hepatocytes cells (PAS+D) and fibrosis; they have shown that accumulation of $P A S+D$ increases with progression of the stage of fibrosis. Consequently, only patients carrying AAT variants able to aggregate are susceptible to liver disease and protein retention without aggregation does not lead to liver disease.

$Z$ aggregates, of liver origin, are also present in the serum of all ZZ patients. Consequently, an association between circulating Z-aggregate concentration and liver fibrosis/liver disease has been suggested. ${ }^{13,14}$

\section{Figure 1. Z-Alpha-1 Antitrypsin Deficiency Inclusion Bodies}

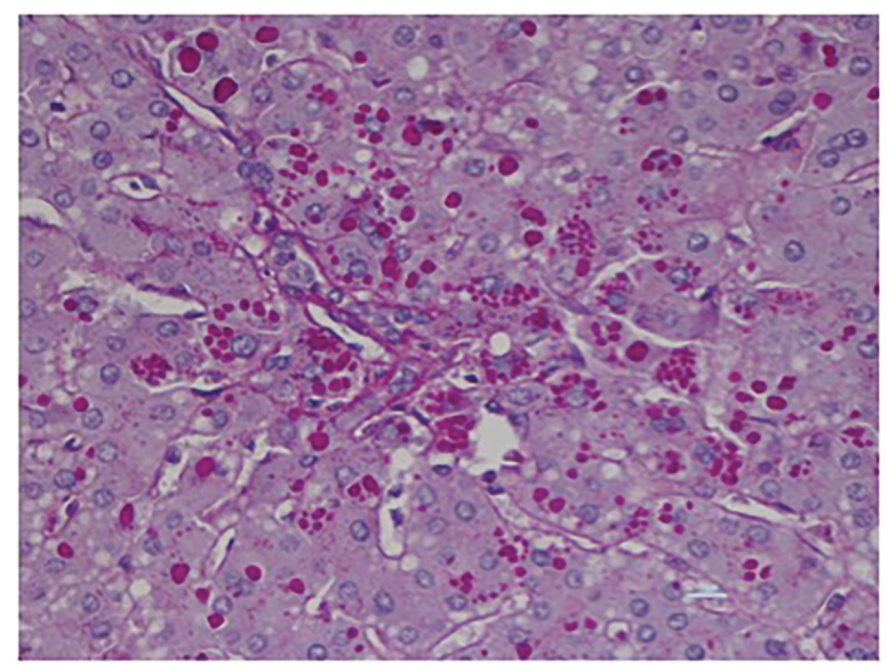

Human ZZ liver stained with hematoxylin-eosin and PAS-Diastase digestion (PAS+D). The PAS-Diastase stains glycoproteins and consequently IBs in red. There is heterogeneity in the distribution and size of IBs within hepatocytes, many are free or with small "dust" of IBs and others with huge and well-marked IBs.

PAS=periodic acid-Schiff; IBs=inclusion bodies

Nevertheless, this association requires further studies to establish whether this biomarker could be useful as a prognostic factor in disease outcomes.

The Z-variant is not the only AAT mutant involved in liver diseases. Rare variants, such as the Mmalton, (Phe51/52 del) and Siiyama (Ser53Phe) variants, may also cause liver disease. ${ }^{15,16}$ Similar to the $\mathrm{Z}$ mutant, these rare variants are able to form aggregates and IBs in the hepatocytes. However, some data have suggested that the process and/or the nature of aggregates involved in these mutants, Z, Mmalton and Siiyama variants, are different. ${ }^{17,18}$

There is no specific treatment for AATD-associated liver disease, only the standard liver supportive care, such as ursodeoxycholic acid, is used in pediatric practice. ${ }^{19}$ In general, AATD patients should receive hepatitis vaccinations to prevent further liver injury, follow nutritional management and recommendations and avoid alcohol intake to prevent fatty liver disease and/or obesity. ${ }^{20}$ However, in severe AATD-related liver disease (liver failure, decompensated cirrhosis), when the disease becomes life-threatening, a liver transplant is performed with excellent survival rates: $90 \%$ at 1 year and $80 \%$ at 5 years. ${ }^{21}$

Although the $Z$ protein accumulation is the 
primary cause of liver damage, this event is not sufficient. Indeed, not all homozygous ZZ patients develop liver injury despite the presence of IBs in the liver. $^{22,23}$ Therefore, there is likely a significant role for second hits such as genetic and/or environmental modifiers, ${ }^{12,24,25}$ which determine whether an AATD patient will or will not develop liver injury.

The natural history of AATD is not well defined and liver disease is a lifelong condition. ${ }^{26,27}$ However, AATD-related liver disease has been described as being different at different stages of life. In childhood, AATD is the most common genetic cause of pediatric liver disease and the most frequent inherited indication for liver transplantation in the pediatric population. ${ }^{28}$ Over the past 20 years, there has been an increase in the prevalence of the adult form of AATD-mediated liver disease. AATD-related liver disease displays a biphasic pattern with the first peak in an early childhood (birth to 5 years) and the second peak, in adulthood between 50-65 years of age. ${ }^{26}$ The adult form of the disease seems to be an age-dependent degenerative disease contrary to the pediatric form of the disease, whose severity seems more associated with genetic factors. ${ }^{26,29}$ Thus, classic confounding factors encountered in childhood, such as breast feeding or other liver diseases (glycogen storage disease or cystic fibrosis), have not been associated so far with this severity. ${ }^{29}$

In this review, we pinpoint the main, known cofactors of liver disease in AATD in childhood and adulthood.

\section{Why Do Some Patients Do Poorly During Childhood?}

Large pediatric cohorts have been investigated for factors responsible for liver disease and have shown that the natural history of AATD is highly variable, ranging from mild elevations in liver blood tests to liver failure necessitating liver transplantation. ${ }^{23,29-31}$

The main clinical characteristic is usually a prolonged cholestatic jaundice during the first 2 months of life after birth. In an unbiased Swedish study, the authors screened 200,000 newborns in Sweden and detected 127 homozygous ZZ patients in the 1970s and concluded then that only $10 \%$ of homozygous ZZ patients develop clinically significant liver disease in the first 4 decades of life and $5 \%$ of them have the risk of life-threatening liver disease ${ }^{31,32}$ (Figure 2). In addition to this pioneer and massive population-based study, more recently the French longitudinal study named DEFI-ALPHA that included only pediatric patients with known AATD irrespective of genotype, observed $18.3 \%$ of pediatric AATD patients had a severe liver disease (portal hypertension, presence of oesophageal varices, liver failure, liver transplantation or liver-related death) and almost half of them ended up with a liver transplantation. ${ }^{29}$ As supported by other works, the authors have observed that neonatal cholestasis at diagnosis was significantly associated with severe liver disease. Other signs have also been correlated with worse prognosis such as persistence of elevated liver enzyme, hepatomegaly, and early development of splenomegaly. ${ }^{30,33}$

\section{Figure 2. Risk Factors Predisposing to a Significant Liver Injury in Children and Adults}

\section{Pediatric Patients at Risk}

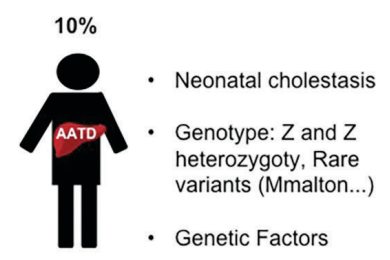

\section{Adult Patients at Risk}

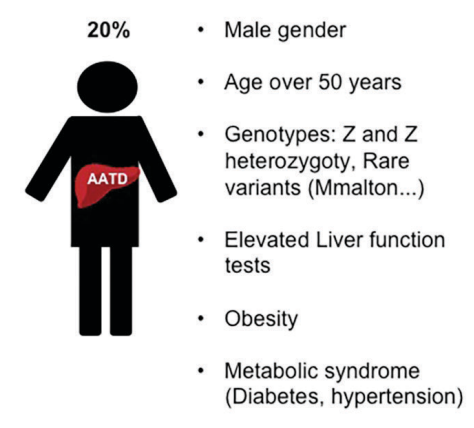

Although a small proportion of infants will develop severe liver injury, the majority of individuals will have their liver-related parameters frequently improve; $80 \%$ of cholestatic AATD infants will have resolution of the cholestasis and develop normally without clinical evidence of chronic liver disease or presence of advanced liver disease. ${ }^{23,28,34}$

From the French study, we also learned that liver disease is not restricted to ZZ genotypes; individuals with other genotypes, such as SZ and MMalton variants, may also develop liver damage, especially when associated with other predisposing factors (glycogen storage disease or cystic fibrosis) (Figure 2). Those diseases are already known to be worsened by AATD variants, for instance MZ genotype was shown 
to aggravate liver disease severity in children with cystic fibrosis. ${ }^{32}$ However, the inverse is not true, no genetic polymorphisms in CFTR or SERPINA 1 genes influence the onset and severity of liver disease in AATD during childhood. ${ }^{35}$

It is still unknown why patients with the same genotype, even siblings, have such varied clinical courses. It is now well recognized that environmental and/or genetic modifiers, yet to be fully elucidated, are involved in these clinical features. To date, 2 genetic studies, a candidate gene-sequencing strategy ${ }^{24}$ and a genome-wide association study ${ }^{25}$ have highlighted some potential genetic modifiers and pinpointed the ERAD pathway as an important player in AATD-related liver disease. Indeed, in 2009, Pan et al demonstrated that difference in ER mannosidase I (ERmanI) expression, a central component of ER quality control, ERAD and consequently, in AAT degradation, was associated with an earlier age-of-onset for endstage liver disease. ${ }^{24}$ Recently, our group identified that HERPUD 1 R5OH and HFE H63D variants are associated with the advanced liver disease component of AATD. ${ }^{25}$ We also observed that specific pathways, including ERAD and unfolded protein response (UPR), may be risk factors for AATD-caused liver disease. ${ }^{25}$

However, many other factors are likely to be able to modify AATD-associated liver disease and must be elucidated. Several publications suggest that genetic factors affecting the efficiency of the ERAD/UPS and/or autophagy might act as potential modifiers of AATD- liver disease. There is evidence suggesting that Z-AAT degradation is significantly slower in cells from AATD patients with liver disease than in cells from ATD patients without liver disease. ${ }^{36-38}$ This suggests that disposal pathways (ERAD/UPS, autophagy) seem relatively inefficient in $\mathrm{ZZ}$ individuals presenting liver disease phenotype. Further genome-wide association studies may allow us to explore and resolve the role of these pathways and more importantly identify those genetic modifiers in the future.

\section{Why Do Some Patients Do Poorly During Adulthood?}

Liver disease in adults is much more frequent than previously thought. Following a Swedish postmortem study published by Eriksson, 5\%-10\% of the ZZ individuals over 50 years of age had developed cirrhosis. ${ }^{9}$ Similarly, long-term natural history studies of liver disease in AATD have recently shown the prevalence of significant liver fibrosis $(F \geq 2)$ in $\mathrm{ZZ}$ adults is about $30 \%^{12,39}$ and the prevalence of advanced liver disease (cirrhosis, hepatocellular carcinoma) is $10 \%$ (Figure 2). ${ }^{40}$ From 1991 to 2012 , $77.2 \%$ of transplants for AATD were in adults, and this increased to $87.4 \%$ in the last 10 years, suggesting that liver disease is more common in adults than in children. Nevertheless, liver disease in adults is still under-recognized and undiagnosed because there are no specific biochemical and histopathologic markers and analyses to state about AATD-liver disease. All AATD adults should be tested and screened for liver disease given that one-third of adults with $\mathrm{ZZ}$ genotype have significant fibrosis without clinical liver disease $^{12}$ (Figure 2). It is now clear that in $\mathrm{ZZ}$ adult males, elevated liver enzymes (upper limit of normal), metabolic syndromes (diabetes) and increasing body mass index (BMI) were identified as risk factors for the development of end-stage liver disease requiring transplantation (Figure 2). ${ }^{12,27,40,41}$ The association between obesity and AATD was recently confirmed at the cellular level by comparing AATD epigenetic changes to those linked to liver disease driven by other etiologic exposures (alcohol use, viral hepatitis). AATD and obesity-driven liver disease have marked overlap. $^{42}$

In contrast, the other classic liver disease co-factors, such as viral hepatitis, autoimmune hepatitis, iron overload, alcohol use or cholestatic liver diseases, and their association with AATD liver disease are still debated. All these co-factors have not been confirmed in all studies. ${ }^{12,27,40,41}$ Nevertheless, the $Z$ allele may act as a risk factor for cirrhosis in the pathogenesis process of alcoholic and non-alcoholic fatty liver diseases. ${ }^{43,44}$ Recently, it has also been shown that ZZ carriers had lower serum concentrations of triglyceride, and low and very low-density lipoprotein cholesterol than controls. These results suggest that Z-AATD patients have impaired hepatic secretion of lipids. ${ }^{39}$ Finally, no statistically significant relationship between the presence of liver fibrosis and severity of COPD was found. ${ }^{12,27,40,41}$

Interestingly, individuals with liver disease in the neonatal period and who survived childhood do not suffer from liver disease in adulthood. Consequently, liver disease in childhood seems not to directly influence the risk of liver disease in adults. ${ }^{40,45}$

As observed for the pediatric forms, specific rare 
AATD (Mmalton) and ZZ and heterozygous $Z$ types are associated with liver fibrosis development, suggesting that the $\mathrm{Z}$ variant is a clear and strong disease modifier. Hence, it has been suggested that heterozygosis increases the risk of developing liver disease. The incidence of liver disease could be higher in heterozygotes with the deficiency than in the general population, especially if the affected individuals have other liver comorbidities. ${ }^{5}$ Many patients who undergo liver transplantation with a diagnosis of AATD are actually heterozygotes who also have other risk factors (alcohol consumption, steatosis). ${ }^{26,46}$

Chronic liver disease is a major risk factor for the development of liver cancer. The 2 most common forms of primary liver cancer are hepatocellular carcinoma (HCC) and cholangiocarcinoma (bile duct cancer). AATD patients are more likely to develop HCC than cholangiocarcinoma (AATD-mediated cholangiocarcinoma has been reported very rarely). Compared to childhood, in which liver cancer (especially HCC) is reported as extremely rarely, in adults, a post mortem examination has shown that $28 \%$ of ZZ individuals had HCC. ${ }^{9}$ Recently, a longitudinal follow-up of liver function and occurrence of liver disease in $1595 \mathrm{ZZ}$ individuals has found HCC in $2 \%$ (29) of the individuals. The overall incidence is $1.3 \%$ and this proportion is similar for other liver etiologies such as alcohol. ${ }^{47}$ Moreover, more than $90 \%$ of cases of HCC occur in patients with cirrhosis. Concerning AATD-mediated HCC, in the 29 patients with HCC, the majority (16 patients) had $\mathrm{HCC}$ as a primary diagnosis. ${ }^{40}$ Consequently, $\mathrm{ZZ}$ individuals with liver disease are prone to develop HCC and it can occur without preceding cirrhosis. ${ }^{40,48}$

\section{Why Do Some Patients Do Poorly from a Cellular Pathophysiology Perspective?}

Although all the molecular mechanisms underlying hepatocyte damage are not fully understood, we already know that the accumulation of $\mathrm{Z}$ aggregates will trigger a cascade of stress pathways that will lead to the death of the hepatocyte and liver injury (chronic hepatitis, cirrhosis, and HCC) (Figure 3). Indeed, the apoptosis pathway is induced potentially through mitochondrial dysfunction. ${ }^{49}$ The induction of this death pathway is closely associated with the accumulation of the $Z$ variant and has an important

\section{Figure 3. Cellular Mechanisms Involved in Injury Cascade of Z-Alpha Antitrypsin Mutant in Liver}

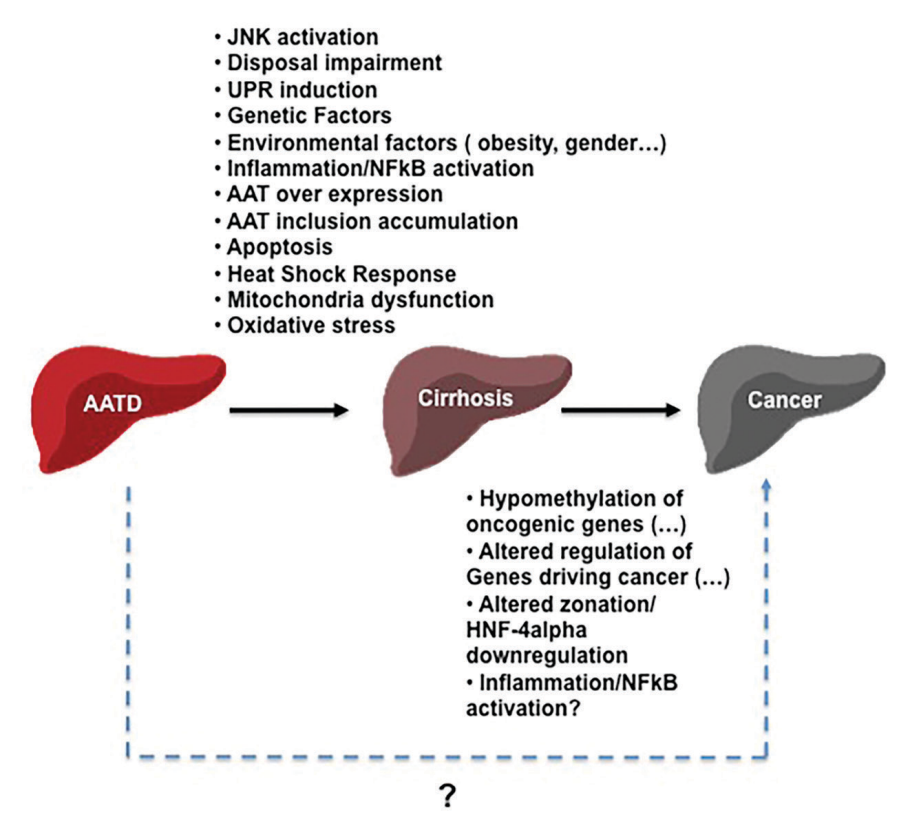

$\mathrm{JNK}=$ jun-N-terminal kinase; UPR=unfolded protein response; $\mathrm{AAT}=$ alpha- 1 antitrypsin; HNF-4alpha=hepatocyte nuclear factor 4 alpha

impact on the liver disease process. Hepatocytes expressing the $Z$ aggregates have an increased caspase-activated mitochondrial depolarization and are more prone to apoptosis (Figure 3). ${ }^{50}$ Therefore, the hepatocytes containing Z IBs are impaired in cell proliferation compared to the cells without or with lesser $\mathrm{Z}$ accumulation/IB which are promoted to proliferate to preserve the liver cell mass. In a vicious cycle of death and regeneration, the hepatic stellate cells are activated which initiates the hepatic fibrosis process. This hypothesis was also supported by experiments in which normal hepatocytes were found to have a selective proliferative advantage in the liver when transplanted into a AATD mouse model. ${ }^{51}$

Activation of NFkB is another hallmark of the cellular response to $Z$ accumulation (Figure 3). Little is known about the mechanism underlying the activation of this pathway but it was suggested that ER morphological changes caused by the accumulation of $Z$ aggregates might disrupt ER calcium homeostasis leading to release into the cytosol and NFkB activation. ${ }^{52-54}$ In a mouse model, it was also observed that NFkB activation could prevent fibrosis associated with 
Z-AATD. Z accumulation and hepatic fibrosis were worse in the Z-AATD mouse model crossed with 2 different mice models deficient in NFkB signaling. ${ }^{11,55}$ The regulation of NFkB signaling on autophagy might explain its protective role in the liver fibrosis.

In contrast to the NFkB pathway activation, $\mathrm{Z}$ mutation and/or aggregations fail to induce the UPR, a stress response that controls ER homeostasis through transcriptional activation of several genes involved in ER folding, degradation and export. ${ }^{54}$ Nevertheless, $\mathrm{Z}$ accumulation seems to sensitize the cell to second insults that can cause a much stronger UPR response than observed into WT cells (Figure 3). ${ }^{25,54}$

Curiously, another well-known pathway, induced following protein aggregation- the heat shock response (HSR) pathway- is activated in cells expressing the $\mathrm{Z}$ mutant. The down-regulation of this stress response, through silencing of HSF1, the master regulator of the HSR, is increased maturation and secretion of the mutant Z-AAT. ${ }^{56}$ The mechanism by which the silencing of HSF 1 could be beneficial for AATD still needs to be elucidated but these results suggest a link, a cross talk between the ER compartment and the cytoplasmic stress management by HSR. ${ }^{56}$

Other cellular pathways such as the oxidative stress or c-Jun N-terminal kinase (JNK) and c-Jun signaling (Figure 3) are also induced in response to $\mathrm{Z}$ accumulation. ${ }^{57,58}$ The oxidative stress pathway has been shown to be a contributing factor in the development of liver damage in animal models of AATD. Higher levels of reactive oxygen species and a more oxidized, cellular redox state were observed in liver tissue from AATD mice as compared to WT mice. ${ }^{58}$ JNK signaling was also found to be involved in the development of liver damage. JNK signaling, through the transcription factor c-Jun, is involved in transcriptional regulation of SERPINA 1 and inhibition of JNK was found to reduce $Z$ accumulation. ${ }^{57}$

Environmental factors modulating SERPINA 1 expression may also potentially affect liver injury. AAT is an acute-phase protein that overexpresses (by 2to 4 -folds) in response to inflammatory induction. ${ }^{59}$ This overexpression could consequently increase the amount of $Z$ aggregate forms and increase the burden of Z-AAT hepatotoxicity. In agreement, treatment with indomethacin, a nonsteroidal anti-inflammatory drug, in an AATD mouse model was found to up-regulate AAT expression and enhance liver injury. ${ }^{60}$

Finally, the pathogenesis involved in liver cancer development is still unclear and needs to be elucidated (Figure 3). Nevertheless, some events described by different groups might play a role in carcinogenesis (Figure 3). In an AATD mouse model, a downregulation of hepatocyte nuclear factor 4 alpha (HNF4a) has been observed to be associated with a loss of zonation. ${ }^{61}$ Given that HNF-4a is a master transcription factor in hepatocyte phenotype maintenance ${ }^{62}$ and has a suppressor role in liver cancer, ${ }^{63}$ its downregulation could favor HCC development. Others have also observed a significant genomic hypomethylation in AATD liver-impacting genes related to liver cancer following a DNA methylation study on liver biopsies of 118 ZZ-AATD adult patients (104 without cirrhosis, 14 cirrhotic). ${ }^{42}$ This up-regulation of markers related to liver cancer was also described by Segeritz et al. Based on a comparative "omics" approach, the authors have observed an up-regulation of specific proteins associated with predisposition to malignancy in human ZZ-induced pluripotent stem cells. ${ }^{64}$ Finally, $\mathrm{NF} \mathrm{kB}$ activation-mediated liver inflammation might also play a role in carcinogenesis. ${ }^{65}$

\section{Conclusion}

AATD-mediated liver damage has been linked to a proteotoxicity resulting in the accumulation of misfolded AAT in the ER of hepatocytes triggering a cascade of stress responses and eventually, liver cell death. There is a high degree of clinical variability in the disease outcome and so far, we have not been able to screen individuals at risk of developing liver damage. Nevertheless, recent long-term natural history studies of liver disease in AATD have given insights into AATD-mediated liver damage and some clues to answer the questions raised in the manuscript's title: why do some patients do poorly? what do we know so far?

AATD-related liver disease displays a biphasic pattern with the first peak in early childhood (birth to 5 years) and the second peak, in adulthood between $50-65$ years of age. ${ }^{26}$

Beginning in childhood, only $10 \%$ of homozygous ZZ patients develop clinically significant liver disease in the first 4 decades of life. ${ }^{31,32}$ Neonatal cholestasis at diagnosis is significantly associated with severe liver disease (Figure 2). Liver disease is not restricted to ZZ genotypes; individuals with other genotypes such as SZ 
and Mmalton variants may also develop liver damage (Figure 2). Even so, it is still unknown why patients with the same genotype, even siblings, have such varied liver clinical courses. It is now well recognized that environmental and/or genetic modifiers, yet to be fully elucidated, are involved in these clinical features. To date, 2 genetic studies ${ }^{24,25}$ have highlighted some potential genetic modifiers and pinpointed the ERAD pathway as an important player in AATD-related liver disease (Figure 3).

In adulthood, AATD-liver disease is a much more frequent occurrence than previously appreciated. It is now clear that in $\mathrm{ZZ}$ adult males over 50 years of age, elevated liver enzymes (upper limit of normal), metabolic syndromes (diabetes) and increasing BMIs are identified as risk factors for the development of endstage liver disease requiring transplantation (Figure 2). ${ }^{12,27,40,41}$ Finally, as observed for the pediatric forms, rare AATD genotypes such as Mmalton and heterozygous $\mathrm{Z}$ are associated with liver fibrosis development (Figure 2).

Consequently, given all this information, for any child with neonatal cholestasis, a diagnosis of AATD must be considered. Similarly, adult males over 50 with obesity and metabolic syndromes should be tested. The observed commonalities between liver disease and obesity, for instance, suggests that all the patricians should advise patients to lose weight, eat healthy, and avoid alcohol intake and recommend immunization against hepatitis in order to prevent the development of end-stage liver disease in later adulthood.
Clearly these findings have increased our understanding of the disease, nevertheless, many challenges still need to be addressed and consequently, further research is required to identify potential disease-genetic modifiers, unknown factors that lead to increased susceptibility to HCC and molecular mechanisms involved in the disease pathogenesis. These challenges need to be resolved for the development of early and non-invasive diagnosis, and to develop new therapeutic strategies. Indeed, in contrast to the lung disease, for which an augmentation therapy is available for treating frequent and severe pulmonary exacerbations, there are currently no therapies for AATD-associated liver disease beyond transplantation. ${ }^{66}$ Nevertheless, autophagy enhancers, small molecule chaperones, gene repair (CrisprCas9) associated with cell transplantation and siRNA technologies hold promise for the future treatment of this disease. ${ }^{66}$

\section{Acknowledgements}

Grant support was provided by the Association Nationale Francaise Deficit en Alpha-1 Antitrysine, Alpha-1 Association Suisse CSL Behring, the University of Bordeaux, the National Institute of Health and Medical Research (INSERM, and the National Center for Scientific Research (CNRS).

\section{Declaration of Interest}

MB declares no conflicts of interest. 


\section{References}

1. Laurell CB, Eriksson S. The electrophoretic alpha1-globulin pattern of serum in alpha- 1 antitrypsin deficiency. Scand J Clin Lab Invest. 1963;15(2):132-140.

2. Bouchecareilh M, Conkright JJ, Balch WE. Proteostasis strategies for restoring alpha-1 antitrypsin deficiency. Proc Am Thorac Soc. 2010;7(6);415-422.

doi: https://doi.org/10.1513/pats.201001-016AW

3. Bouchecareilh M. [Alpha-1 antitrypsin deficiency]. Med Sci (Paris). 2014; 30(10): 889-895.

doi: https://doi.org/10.1051/medsci/20143010016

4. Salahuddin, P. Genetic variants of alpha-1 antitrypsin. Curr Protein Pept Sci. 2010; 11(2):101-117.

doi: https://doi.org/10.2174/138920310790848368

5. Laurell CB. Genetic variants of alpha-1 antitrypsin. Vox Sang. 1965;10:363-364.

6. Greene CM, Marchiniak SJ, Teckman J, et al. Alpha-1 antitrypsin deficiency. Nat Rev Dis Primers. 2016; 2:16051.

doi: https://doi.org/10.1038/nrdp.2016.51

7. Kruse KB, Brodsky JL, McCracken AA. Characterization of an ERAD gene as VPS30/ATG6 reveals two alternative and functionally distinct protein quality control pathways: one for soluble $\mathrm{Z}$ variant of human alpha-1 proteinase inhibitor (A1PiZ) and another for aggregates of A1PiZ. Mol Biol Cell. 2006;17(1):203-212.

doi: https://doi.org/10.1091/mbc.e04-09-0779

8. Haddock CJ, Blomenkemp K, Gautam M, et al. PiZ mouse liver accumulates polyubiquitin conjugates that associate with catalytically active 26 S proteasomes. PLoS One. 2014; 9: e106371. doi: https://doi.org/10.1371/journal.pone.0106371

9. Eriksson S, Carlson J, Velez R. Risk of cirrhosis and primary liver cancer in alpha-1 antitrypsin deficiency. $N$ Engl J Med. 1986; 314:736-739.

doi: https://doi.org/10.1056/NEJM198603203141202

10. Guo S, Booten SL, Aghajan M, et al. Antisense oligonucleotide treatment ameliorates alpha-1 antitrypsin-related liver disease in mice. J Clin Invest. 2014;124:251-261.

doi: https://doi.org/10.1172/JCI67968

11. Hidvegi T, Ewing M, Hale D, et al. An autophagy-enhancing drug promotes degradation of mutant alpha1-antitrypsin $\mathrm{Z}$ and reduces hepatic fibrosis. Science. 2010; 329(5988):229-232. doi: https://doi.org/10.1126/science.1190354

12. Clark VC, Marek G, Liu C, et al. Clinical and histologic features of adults with alpha- 1 antitrypsin deficiency in a non-cirrhotic cohort. J Hepatol. 2018; 69(6):1357-1364. doi: https://doi.org/10.1016/j.jhep.2018.08.005
13. Tan L, Dickens JA, DeMeo DL, et al. Circulating polymers in alpha-1 antitrypsin deficiency. Eur Respir J. 2014; 43(5):15011504. doi: https://doi.org/10.1183/09031936.00111213

14. Janciauskiene S, Wallmark A, Piitulainen E, Kohmlein T, Welte T, Sveger T. Performance of enhanced liver fibrosis plasma markers in asymptomatic individuals with ZZ alpha- 1 antitrypsin deficiency. Eur J Gastroenterol Hepatol. 2011; 23(8):716-720. doi: https://doi.org/10.1097/MEG.0b013e328347daaf

15. Lomas DA, Elliott PR, Sidhar SK, et al. Alpha-1 antitrypsin Mmalton (Phe52-deleted) forms loop-sheet polymers in vivo. Evidence for the C sheet mechanism of polymerization. J Biol Chem.1995;270:16864-16870. doi: https://doi.org/10.1074/jbc.270.28.16864

16. Lomas DA, Finch JT, Seyama K, Nukiwa T, Carrell RW. Alpha-1 antitrypsin Siiyama (Ser53->Phe). Further evidence for intracellular loop-sheet polymerization. J Biol Chem.1993; 268:15333-15335.

17. Janciauskiene S, Eriksson S, Callea F, et al. Differential detection of PAS-positive inclusions formed by the Z, Siiyama, and Mmalton variants of alpha-1 antitrypsin. Hepatology. 2004; 40(5):1203-1210. doi: https://doi.org/10.1002/hep.20451

18. Callea F, Giovannoni I, Francalanci P, et al. Mineralization of alpha-1-antitrypsin inclusion bodies in Mmalton alpha-1 antitrypsin deficiency. Orphanet J Rare Dis. 2018;13:79. doi: https://doi.org/10.1186/s13023-018-0821-7

19. Teckman JH. Liver disease in alpha-1 antitrypsin deficiency: current understanding and future therapy. COPD. 2013;10 (Suppl 1):35-43. doi: https://doi.org/10.3109/15412555.2013.765839

20. European Association for the Study of the Liver. EASL clinical practice guidelines on nutrition in chronic liver disease. $J$ Hepatol. 2019;70(1):172-193. doi: https://doi.org/10.1016/j.jhep.2018.06.024 .

21. Kayler LK, Merion RM, Lee S, et al. Long-term survival after liver transplantation in children with metabolic disorders. Pediatr Transplant. 2002;6(4):295-300. doi: https://doi.org/10.1034/j.1399-3046.2002.02009.x

22. Teckman JH, Mangalat N. Alpha-1 antitrypsin and liver disease: mechanisms of injury and novel interventions. Expert Rev Gastroenterol Hepatol. 2015;9:261-268.

doi: https://doi.org/10.1586/17474124.2014.943187

23. Teckman JH, Rosenthal P, Abel R, et al. Baseline analysis of a young alpha-1 antitrypsin deficiency liver disease cohort reveals frequent portal hypertension. J Pediatr Gastroenterol Nutr. 2015;61(1):94-101.

24. Pan S, Huang L, McPherson J, et al. Single nucleotide polymorphism-mediated translational suppression of endoplasmic reticulum mannosidase I modifies the onset of endstage liver disease in alpha-1 antitrypsin deficiency. Hepatology. 2009;50(1):275-281. doi: https://doi.org/10.1002/hep.22974 
25. Joly P, Vignaud H, DiMartino J, et al. ERAD defects and the HFEH63D variant are associated with increased risk of liver damages in alpha-1 antitrypsin deficiency. PLoS One. 2017;12:e0179369. doi: https://doi.org/10.1371/journal.pone.0179369

26. Chu AS, Chopra KB, Perlmutter, DH. Is severe progressive liver disease caused by alpha-1 antitrypsin deficiency more common in children or adults? Liver Transpl. 2016;22(7):886-894. doi: https://doi.org/10.1002/lt.24434

27. Townsend SA, Edgar RG, Ellis PR, Kantas D, Newsome PN, Turner AM. Systematic review: the natural history of alpha-1 antitrypsin deficiency, and associated liver disease. Aliment Pharmacol Ther. 2018;47(7):877-885.

doi: https://doi.org/10.1111/apt.14537

28. Feldman A, Sokol RJ. Alpha-1 antitrypsin deficiency: an important cause of pediatric liver disease. Lung Health Prof Mag. 2013;4:8-11.

29. Ruiz M, Lacaille R, Berthella J, et al. Liver disease related to alpha1-antitrypsin deficiency in French children: The DEFIALPHA cohort. Liver Int. 2019;39(6):1136-1146. doi: https://doi.org/10.1111/liv.14035

30. Ibarguen E, Gross CR, Savik SK, Sharp HL. Liver disease in alpha-1 antitrypsin deficiency: prognostic indicators. J Pediatr. 1990;117(6):864-870.

doi: https://doi.org/10.1016/S0022-3476(05)80123-4

31. Sveger T. Liver disease in alpha-1 antitrypsin deficiency detected by screening of 200,000 infants. $N$ Engl J Med.1976; 294:13161321. doi: https://doi.org/10.1056/NEJM197606102942404

32. Sveger T. The natural history of liver disease in alpha-1 antitrypsin deficient children. Acta Paediatr Scand. 1988;77(6):847-851. doi: https://doi.org/10.1111/j.1651-2227.1988.tb10767.x

33. Nebbia G, Hadchouel M, Odievre M, Alagille D. Early assessment of evolution of liver disease associated with alpha-1 antitrypsin deficiency in childhood. J Pediatr. 1983;102(5):661-665. doi: https://doi.org/10.1016/S0022-3476(83)80230-3

34. Sveger T, Eriksson, S. The liver in adolescents with alpha-1 antitrypsin deficiency. Hepatology.1995;22(2):514-517. doi: https://doi.org/10.1016/0270-9139(95)90573-1

35. Joly P, Lachaux A, Ruiz M, et al. SERPINA1 and MAN1B1 polymorphisms are not linked to severe liver disease in a French cohort of alpha-1 antitrypsin deficiency children. Liver Int. 2017;37(11):1608-1611. doi: https://doi.org/10.1111/liv.13586

36. Wu Y, Whitman I, Molmenti E, Moore K, Hippenmeyer P, Perlmutter DH. A lag in intracellular degradation of mutant alpha 1-antitrypsin correlates with the liver disease phenotype in homozygous PiZZ alpha 1-antitrypsin deficiency. Proc Natl Acad Sci U S A. 1994; 91(19):9014-9018. doi: https://doi.org/10.1073/pnas.91.19.9014
37. Teckman JH, Perlmutter DH. The endoplasmic reticulum degradation pathway for mutant secretory proteins alphalantitrypsin $\mathrm{Z}$ and $\mathrm{S}$ is distinct from that for an unassembled membrane protein. J Biol Chem. 1996; 271:13215-13220. doi: https://doi.org/10.1074/jbc.271.22.13215

38. Tafaleng EN, Chakraborty S, Han B, et al. Induced pluripotent stem cells model personalized variations in liver disease resulting from alpha-1 antitrypsin deficiency. Hepatology. 2015;62(1):147-157. doi: https://doi.org/10.1002/hep.27753

39. Hamesch K, Mandorfer M, Pereira VM, et al. Liver fibrosis and metabolic alterations in adults with alpha-1 antitrypsin deficiency caused by the PI*ZZ mutation. Gastroenterology. 2019;157(3):705-719.

40. Tanash HA, Piitulainen E. Liver disease in adults with severe alpha-1 antitrypsin deficiency. J Gastroenterol. 2019; 54:541-548. doi: https://doi.org/10.1007/s00535-019-01548-y

41. Bowlus CL, Willner I, Zern MA, et al. Factors associated with advanced liver disease in adults with alpha-1antitrypsin deficiency. Clin Gastroenterol Hepatol. 2005;3(4):390-396. doi: https://doi.org/10.1016/S1542-3565(05)00082-0

42. Wang L, Marek GW III, Hlady RA, et al. Alpha-1 antitrypsin deficiency liver disease, mutational homogeneity modulated by epigenetic heterogeneity with links to obesity. Hepatology. 2019;70(1): 51-66. doi: https://doi.org/10.1002/hep.30526

43. El-Rayah EA, Twomey PJ, Wallace EM, McCormick PA. Both alpha-1 antitrypsin Z phenotypes and low caeruloplasmin levels are over-represented in alcohol and nonalcoholic fatty liver disease cirrhotic patients undergoing liver transplant in Ireland. Eur J Gastroenterol Hepatol. 2018;30(4):364-367. doi: https://doi.org/10.1097/MEG.0000000000001056

44. Strnad P, Buch S, Hamesch J, et al. Heterozygous carriage of the alpha-1 antitrypsin $\mathrm{Pi}^{*} \mathrm{Z}$ variant increases the risk to develop liver cirrhosis. Gut. 2018;56(1): E2-E89. doi: https://doi.org/10.1055/s-0037-1612696

45. Mostafavi B, Diaz S, Tanash HA, Piitulainen E. Liver function in alpha- 1 antitrypsin deficient individuals at 37 to 40 years of age. Medicine (Baltimore). 2017;96:e6180. doi: https://doi.org/10.1097/MD.0000000000006180

46. Perlmutter DH. Current and emerging treatments for alpha-1 antitrypsin deficiency. Gastroenterol Hepatol (N Y). 2016;12(7):446-448.

47. Fattovich G, Stroffolini T, Zagni I, Donato F. Hepatocellular carcinoma in cirrhosis: incidence and risk factors. Gastroenterology. 2004;127(5 Suppl 1):S35-S50. doi: https://doi.org/10.1053/j.gastro.2004.09.014 
48. Massi G. Pathogenesis and pathology of liver disease associated with alpha-1 antitrypsin deficiency. Chest.1996;110(6 Suppl):251S-255S.

doi: https://doi.org/10.1378/chest.110.6_Supplement.251S

49. Teckman JH, An JK, Blomenkamp K, Schmidt B, Perlmutter D. Mitochondrial autophagy and injury in the liver in alphalantitrypsin deficiency. Am J Physiol Gastrointest Liver Physiol. 2004;286(5):G851-862 (2004).

doi: https://doi.org/10.1152/ajpgi.00175.2003

50. Hidvegi T, Schmidt BZ, Hale P, Perlmutter DH. Accumulation of mutant alpha1-antitrypsin $\mathrm{Z}$ in the endoplasmic reticulum activates caspases- 4 and -12, NFkappaB, and BAP31 but not the unfolded protein response. J Biol Chem. 2005;280:39002-39015. doi: https://doi.org/10.1074/jbc.M508652200

51. Ding J, Yannam GR, Roy-Chowdhury N, et al. Spontaneous hepatic repopulation in transgenic mice expressing mutant human alpha-1 antitrypsin by wild-type donor hepatocytes. $J$ Clin Invest. 2011;121:1930-1934.

doi: https://doi.org/10.1172/JCI45260

52. Carlson JA, Rogers BB, Sifers RN, et al. Accumulation of PiZ alpha-1antitrypsin causes liver damage in transgenic mice. $J$ Clin Invest. 1989;83:1183-1190.

doi: https://doi.org/10.1172/JCI113999

53. Davies MJ, Miranda E, Roussel BD, et al. Neuroserpin polymers activate NF-kappaB by a calcium signaling pathway that is independent of the unfolded protein response. $\mathrm{J}$ Biol Chem. 2009;284; 18202-18209.

doi: https://doi.org/10.1074/jbc.M109.010744

54. Ordonez A, Snapp EL, Tan L, Miranda E, Marciniak SJ, Lomas DA. Endoplasmic reticulum polymers impair luminal protein mobility and sensitize to cellular stress in alpha-1 antitrypsin deficiency. Hepatology. 2013; 57(5):2049-2060

doi: https://doi.org/10.1002/hep.26173

55. Mukherjee A, Hidvegi T, Araya P, Ewing M, Stolz DB, Perlmutter DH. NFkappaB mitigates the pathological effects of misfolded alpha-1 antitrypsin by activating autophagy and an integrated program of proteostasis mechanisms. Cell Death Differ. 2019;26:455-469. doi: https://doi.org/10.1038/s41418-018-0130-7

56. Roth DM, Hutt DM, Tong J, et al. Modulation of the maladaptive stress response to manage diseases of protein folding. PLOS Biol. 2014; 12: e1001998.

doi: https://doi.org/10.1371/journal.pbio.1001998

57. Pastore N, Attanasio S, Granese B, et al. Activation of the c-Jun $\mathrm{N}$-terminal kinase pathway aggravates proteotoxicity of hepatic mutant Z alpha-1 antitrypsin. Hepatology. 2017;65(6):1865-1874. doi: https://doi.org/10.1002/hep.29035
58. Marcus NY, Blomenkamp K, Ahmad M, Teckman JH. Oxidative stress contributes to liver damage in a murine model of alpha-1 antitrypsin deficiency. Exp Biol Med (Maywood). 2012; 237:11631172. doi: https://doi.org/10.1258/ebm.2012.012106

59. Aronsen KF, Ekelund G, Kindmark CO, Laurell CB. Sequential changes of plasma proteins after surgical trauma. Scand J Clin Lab Invest. 1972;29(Suppl 124):127-136. doi: https://doi.org/10.3109/00365517209102760

60. Rudnick DA, Shikapwashya O, Blomenkamp K, Teckman JH. Indomethacin increases liver damage in a murine model of liver injury from alpha-1 antitrypsin deficiency. Hepatology. 2006; 44(4):976-982. doi: https://doi.org/10.1002/hep.21326

61. Piccolo P, Annunziata P, Soria LR, et al. Down-regulation of hepatocyte nuclear factor-4alpha and defective zonation in livers expressing mutant $\mathrm{Z}$ alpha-1 antitrypsin. Hepatology. 2017; 66(1):124-135. doi: https://doi.org/10.1002/hep.29160

62. Hayhurst GP, Lee YH, Lambert G, Ward JM, Gonzalez FJ. Hepatocyte nuclear factor 4alpha (nuclear receptor 2A1) is essential for maintenance of hepatic gene expression and lipid homeostasis. Mol Cell Biol. 2001;21:1393-1403. doi: https://doi.org/10.1128/MCB.21.4.1393-1403.2001

63. Ning B-F, Ping J, Yin C, et al. Hepatocyte nuclear factor 4 alpha suppresses the development of hepatocellular carcinoma. Cancer Res. 2010;70(19):7640-7651. doi: https://doi.org/10.1158/0008-5472.CAN-10-0824

64. Segeritz CP, Rashid ST, de Brito MG, et al. hiPSC hepatocyte model demonstrates the role of unfolded protein response and inflammatory networks in alpha-1 antitrypsin deficiency. $J$ Hepatol. 2018; 69(4):851-860. doi: https://doi.org/10.1016/j.jhep.2018.05.028

65. Hoesel B, Schmid JA. The complexity of NF-kappaB signaling in inflammation and cancer. Mol Cancer. 2013;12:86. doi: https://doi.org/10.1186/1476-4598-12-86

66. Karatas E, DiTommaso S, Dugot-Senant N, Lachaux A, Bouchecareilh M. Overview of alpha-1 antitrypsin deficiencymediated liver disease. Eur Med J Hepatol. 2019;7(1):65-69. 